\title{
Adaptation de la loi de mouvement aux systèmes de positionnement à dynamique élevée
}

\author{
Richard BÉarée a et Pierre-Jean Barre \\ Laboratoire d'Électrotechnique et d'Électronique de Puissance de Lille (L2EP), Équipe commande-activité CEMODYNE \\ (http://cemodyne.lille.ensam.fr), ENSAM, 8 avenue Louis XIV, 59046 Lille Cedex, France
}

Reçu le 15 mars 2007, accepté le 6 juillet 2007

\begin{abstract}
Résumé - L'augmentation des performances des machines de positionnement passe par l'augmentation des accélérations et donc des sollicitations transmises à la structure de la machine. Ces contraintes sont susceptibles d'engendrer des déformations et des vibrations dégradant le suivi de profil ainsi que le positionnement final. Les commandes numériques avancées disposent de différentes formes de loi de mouvement (à jerk limité, polynomiale...) qui ont un effet notable sur le compromis entre la durée du mouvement effectif et la précision attendue. L'objectif de cet article vise à démystifier cet effet en proposant une analyse comparative de l'influence de différents types de lois de mouvement sur les vibrations, ainsi que sur la durée du mouvement d'un axe soumis à un mode de déformation prépondérant.
\end{abstract}

Mots clés : Vibration / déformation élastique / loi de mouvement / génération de commande / système à dynamique élevée

\begin{abstract}
Toward an adaptation of the movement law for high-speed positioning systems. The increase in performances for industrial positioning systems implies the increase in accelerations demands and thus the increase in the stresses transmitted to the mechanical components. These stresses can generate deformations and vibrations degrading the tracking accuracy as well as the positioning accuracy. Modern numerical controllers offer different movement laws (limited jerk, polynomial...), which have a notable effect on the compromise between the effective movement time and the expected accuracy. This paper aims at understanding this effect by proposing a comparative analysis of the influence of various movement laws on the vibrations and on the movement time of an axis submitted to a predominating vibratory mode.
\end{abstract}

Key words: Vibration / elastic deformation / movement law / command generation / high-dynamics system

\section{Introduction}

L'augmentation de la productivité des systèmes de positionnement (machines-outils, manipulateurs cartésiens, portiques,...) passe globalement par la réduction de la durée des mouvements. L'accroissement des demandes dynamiques en termes d'accélération et de vitesse maximale conduit ces systèmes à la limite de leurs possibilités technologiques, invalidant de ce fait l'hypothèse classiquement retenue d'un comportement rigide de la charge à positionner. Les modes de structure deviennent dès lors autant de paramètres sensibles s'exprimant sous la forme

\footnotetext{
a Auteur pour correspondance :

richard.bearee@lille.ensam.fr
}

de déformations et de vibrations, qui limitent les performances et dégradent le système par fatigue.

Une des pistes de solution au problème de flexibilité consiste à élaborer une structure de commande faisant appel à des algorithmes sophistiqués et utilisant des références déterminées spécifiquement pour d'une part, convenir à la physique du système et, d'autre part, atteindre les performances escomptées $[1,2]$. Parmi les principales méthodes envisagées, la plus classiquement utilisée consiste à placer des filtres sur les signaux d'erreur des asservissements de façon à éliminer les fréquences associées aux modes vibratoires. Le principal inconvénient des techniques de filtrage réside dans le déphasage qu'elles introduisent. Ce phénomène de retard peut aller à l'encontre des objectifs visés en nécessitant une diminution 


\section{Nomenclature}

\begin{tabular}{|c|c|}
\hline$A_{\mathrm{m}}$ & Accélération maximale $\left[\mathrm{m} \cdot \mathrm{s}^{-2}\right]$ \\
\hline$F$ & Effort de poussée sur le système $[\mathrm{N}]$ \\
\hline$J_{\mathrm{m}}$ & Jerk maximum $\left[\mathrm{m}_{\mathrm{s}} \mathrm{s}^{-3}\right]$ \\
\hline$S_{\mathrm{m}}$ & Snap maximum $\left[\mathrm{m} \cdot \mathrm{s}^{-4}\right]$ \\
\hline \multirow[t]{2}{*}{$T_{\mathrm{f}}, T_{\mathrm{a}}$} & Durée du mouvement, durée des phases \\
\hline & à accélération non nulle $[\mathrm{s}]$ \\
\hline$T_{\mathrm{n}}$ & Période naturelle d'un mode vibratoire $[\mathrm{s}]$ \\
\hline$V_{\mathrm{m}}$ & Vitesse maximale $\left[\mathrm{m}_{\mathrm{s}} \mathrm{s}^{-1}\right]$ \\
\hline$x_{\text {ref }}$ & Profil de référence en position $[\mathrm{m}]$ \\
\hline$\dot{x}, \ddot{x}$ & Dérivée première et seconde par rapport au temps \\
\hline$\varepsilon$ & Erreur dynamique en position $[\mathrm{m}]$ \\
\hline$\omega_{\mathrm{n}}$ & Pulsation naturelle d'un mode vibratoire $\left[\mathrm{rad} . \mathrm{s}^{-1}\right]$ \\
\hline $\max _{\Delta}(\bullet)$ & Maximum de $\bullet$ sur l'espace $\Delta$ \\
\hline
\end{tabular}

des dynamiques, afin de garantir la stabilité du système asservi. Une autre méthode investiguée dans cet article consiste à agir directement sur la forme de la référence de position, c'est-à-dire sur la loi de mouvement. Intuitivement, le degré d'excitation des modes vibratoires du processus sera directement lié au degré de « douceur » de la loi de mouvement. Aujourd'hui toutes les commandes avancées disposent de différents types de loi (jerk limitée, sinus ou sinus carré d'accélération, polynômes...).

Dans cet article, nous proposons une étude comparative de l'influence de ces différentes lois de mouvement sur le comportement vibratoire ainsi que sur la durée du mouvement d'un axe soumis à un mode vibratoire prépondérant.

Nous attirons l'attention du lecteur sur la terminologie utilisée pour nommer les dérivées successives du mouvement. En effet, si le terme de jerk (dérivée de l'accélération) est désormais courant et accepté par tous, les dérivées d'ordres supérieurs ne disposent pas de dénominations normées. Nous utiliserons une terminologie issue de la biotechnologie, seul domaine à utiliser couramment les dérivées de la position pour qualifier les mouvements humains. Ainsi, la dérivée du jerk est appelée snap [3].

\section{La loi de mouvement}

\subsection{Classification des lois de mouvement}

La loi de mouvement d'un système représente la loi horaire imposée à la géométrie désirée. Avant toute chose, une fonction temporelle candidate au titre de loi de mouvement doit être capable de s'adapter aux variations d'échelles du mouvement, c'est-à-dire être « suffisamment paramétrable» pour décrire toutes les longueurs de déplacement possibles sans dégénérescence excessive de sa forme, donc de ses propriétés.

Nous distinguerons dans la suite deux familles de lois de mouvement que nous appellerons respectivement lois
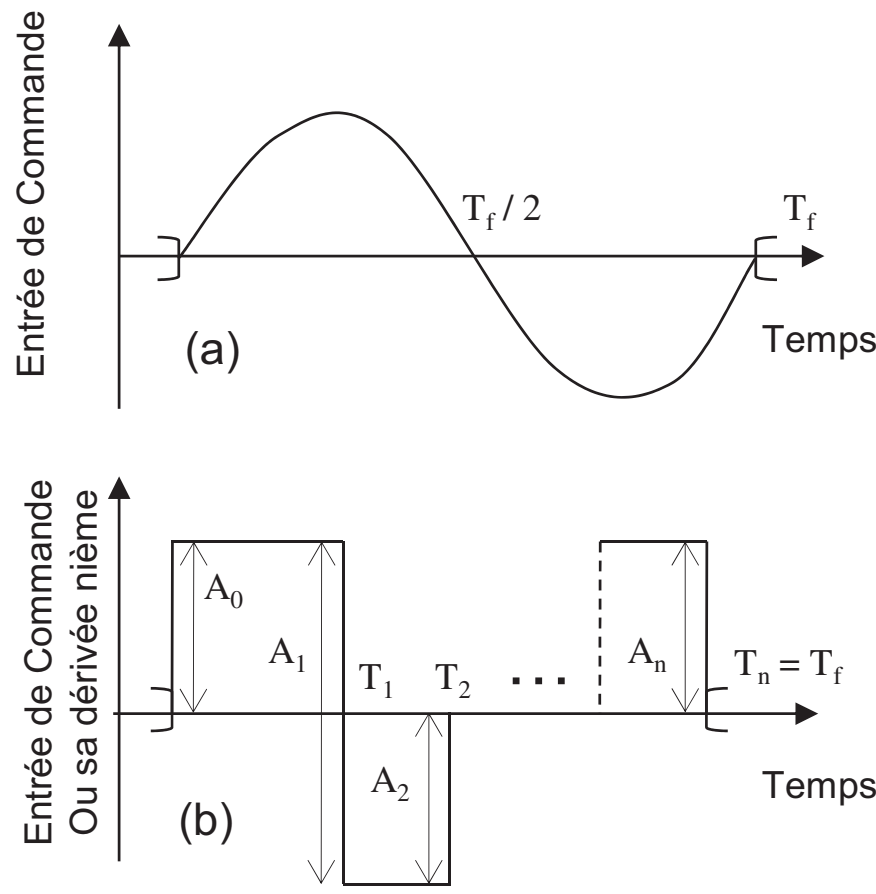

Fig. 1. Forme générique d'une loi de mouvement en accélération : (a) loi douce et (b) loi bang-bang.

douces et lois bang-bang. La figure 1 représente le principe de base différenciant les deux familles de loi de mouvement.

Les lois douces rassemblent toutes les fonctions temporelles dont l'expression analytique vérifie les propriétés nécessaires à l'élaboration d'une loi de mouvement. Bien que l'on puisse imaginer de nombreuses fonctions candidates, les principales fonctions concernées sont les fonctions polynomiales et les fonctions harmoniques. Dans les deux cas, elles permettent de s'adapter aux caractéristiques d'accélération et de décélération; les premières par l'adaptabilité de leur forme (fonction du degré du polynôme) et les dernières par leur périodicité naturelle. Ce type de loi d'une continuité infinie lors du mouvement (en dehors des points extrêmes) aura de façon implicite une plus grande chance de demander un mouvement qui correspond à une trajectoire physique de la machine. Cet effet est généralement englobé sous le qualificatif d'adoucissement de la trajectoire (« smoothing » dans la littérature anglaise) et résulte en une diminution des vibrations durant le mouvement ainsi qu'en une diminution du temps de relaxation du système. Nous verrons que l'inconvénient principal de ces lois est de ne pas être optimales par rapport au temps, contrairement à certaines lois bang-bang. Toutefois, cet inconvénient, i.e. l'augmentation du temps de mouvement théorique, est parfois compensé par la forte diminution du temps de relaxation.

Les lois bang-bang doivent leur appellation à leur principe d'élaboration qui consiste à saturer la variable de commande du système, ou une de ses dérivées, en commutant un certain nombre de fois du niveau maximal au niveau minimal autorisé. La loi de mouvement résultante en position est donc composée d'une succession de courbes 


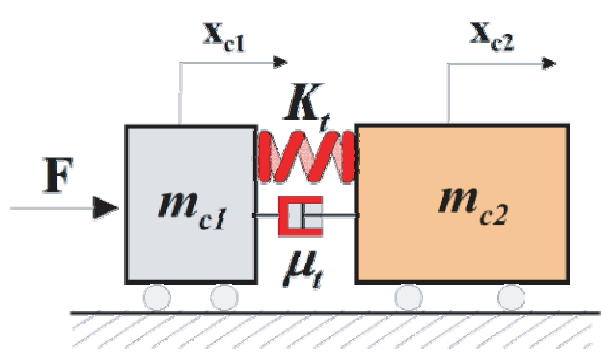

Fig. 2. Modèle d'axe soumis à un mode de déformation prépondérant.

que l'on peut exprimer sous la forme d'une fonction polynomiale par morceaux (spline). La loi de mouvement bang-bang est ainsi entièrement caractérisée par le degré des polynômes utilisés (i.e. l'ordre de la dérivée de la position utilisée), par le nombre de ses polynômes (nombre de commutations moins une), ainsi que par les valeurs des instants de commutation entre chacun des polynômes. Nous verrons que le choix de ces instants de commutation, offre un degré de liberté supplémentaire permettant d'optimiser la loi de mouvement par rapport à certains critères. La plupart des études dédiées à ce type de loi ont porté sur une optimisation par rapport au temps [4]. Mais une technique particulière d'élaboration des lois bangbang, baptisée « input shaping » permet également de les adapter aux caractéristiques vibratoires d'un système [5].

\subsection{Modèle d'étude des vibrations}

Notre objectif consiste à formaliser l'influence de la loi de mouvement sur le mode souple dominant d'un axe. Le modèle d'axe utilisé, décrit à la figure 2, correspond au modèle le plus classiquement usité [6] ; celui d'un axe soumis à un mode de transmission. Les fonctions de transfert canoniques représentant la dynamique d'un tel système entre les vitesses $V_{c 1}$ et $V_{c 2}$ des deux masses et l'effort de commande $F$, s'expriment dans le domaine continu sous la forme :

$$
\begin{gathered}
\frac{V_{c 1}(s)}{F(s)}=\frac{1+\frac{2 \zeta_{n}^{\prime}}{\omega_{n}^{\prime}} s+\frac{1}{\omega_{n}^{\prime \prime}} s^{2}}{m_{\mathrm{tot}} s\left(1+\frac{2 \zeta_{n}}{\omega_{n}} s+\frac{1}{\omega_{n}^{2}} s^{2}\right)} ; \frac{V_{c 2}(s)}{V_{c 1}(s)}=\frac{1+\frac{2 \zeta_{n}^{\prime}}{\omega_{n}^{\prime}} s}{\left(1+\frac{2 \zeta_{n}^{\prime}}{\omega_{n}^{\prime}} s+\frac{1}{\omega_{n}^{\prime 2}} s^{2}\right)} \\
m_{\mathrm{tot}}=m_{c 1}+m_{c 2} ; \quad r=m_{c 2} / m_{c 1} ; \omega_{n}^{\prime}=\sqrt{\frac{K_{t}}{m_{c 2}}} ; \\
\zeta_{n}^{\prime}=\frac{\mu_{\mathrm{t}}}{2 \sqrt{K_{\mathrm{t}} \cdot m_{c 2}}} ; \omega_{n}=\omega_{n}^{\prime} \sqrt{(1+r)} ; \zeta_{n}=\zeta_{n}^{\prime} \sqrt{(1+r)}
\end{gathered}
$$

Dès que la loi de mouvement s'écarte d'une des trajectoires physiques du système, les modes souples sont susceptibles d'être excités et, par conséquent, la précision dynamique est dégradée. Le déplacement effectif de la charge, noté $x_{c 2}$, diffère alors du mouvement idéal. On définit l'erreur dynamique du mouvement :

$$
\varepsilon(t)=x_{\text {ref }}(t)-x_{c 2}(t)
$$

D'après les expressions (1) et (2), en notant que l'accélération de référence est de la forme $\ddot{x}_{\text {ref }}(s)=$ $F(s) / m_{\text {tot }}$, la fonction de transfert régissant l'évolution de l'erreur dynamique dans le cas d'une souplesse dominante de transmission sera donnée par :

$$
\frac{\varepsilon(s)}{x_{\mathrm{ref}}(s)}=\frac{\frac{1}{\omega_{n}^{2}} s^{2}}{1+\frac{2 \zeta_{n}}{\omega_{n}} s+\frac{1}{\omega_{n}^{2}} s^{2}}
$$

Dans la suite, nous nous intéressons aux vibrations induites par la loi de mouvement lors du déplacement, mais également lors de la phase d'arrêt. Ce dernier type de vibration, appelée vibration résiduelle, est un critère dans certains cas prépondérant (exemple du positionnement rapide en robotique, des changements d'outil et phases hors usinage en machine-outil). Pour un système du second ordre, tel que celui représenté par l'erreur dynamique (3), il existe une relation analytique simple permettant de déterminer l'amplitude maximale de l'erreur vibratoire résiduelle en fonction de l'erreur dynamique et de sa dérivée à l'instant final $T_{\mathrm{f}}$. En effet, la résolution de l'équation différentielle donnée par la relation (3) en régime libre $\left(t \geqslant T_{\mathrm{f}} \Rightarrow \ddot{x}_{\text {ref }}(t)=\dot{x}_{\text {ref }}(t)=0\right)$ conduit à l'expression :

$$
\begin{aligned}
\varepsilon(t)= & e^{-\zeta_{n} \omega_{n}\left(t-T_{\mathrm{f}}\right)}\left[C_{1} \cos \left(\omega_{n}\left(t-T_{\mathrm{f}}\right)\right)\right. \\
& \left.+C_{2} \sin \left(\omega_{n}\left(t-T_{\mathrm{f}}\right)\right)\right] \quad \text { pour } t \geqslant T_{\mathrm{f}} \\
\text { avec } \quad C_{1}= & \varepsilon\left(T_{\mathrm{f}}\right) \text { et } C_{2}=\dot{\varepsilon}\left(T_{\mathrm{f}}\right) / \omega_{n}-\varepsilon\left(T_{\mathrm{f}}\right) \zeta_{n} \omega_{n}
\end{aligned}
$$

d'où l'on déduit l'amplitude maximale des vibrations résiduelles :

$$
\begin{aligned}
\max _{t \geqslant T_{\mathrm{f}}}(\varepsilon(t))= & \sqrt{\left(\varepsilon\left(T_{\mathrm{f}}\right)\right)^{2}+\left(\frac{\dot{\varepsilon}\left(T_{\mathrm{f}}\right)}{\omega_{n}}\right)^{2}+\zeta_{n}^{2}\left(\varepsilon\left(T_{\mathrm{f}}\right)\right)^{2}-\frac{2 \zeta_{n} \dot{\varepsilon}\left(T_{\mathrm{f}}\right) \varepsilon\left(T_{\mathrm{f}}\right)}{\omega_{n}}}
\end{aligned}
$$

Afin de simplifier l'analyse comparative des différentes lois de mouvement, nous utilisons une représentation baptisée graphe de vibration. Ce graphe est un diagramme adimensionné représentant l'amplitude maximale des vibrations (lors du mouvement et résiduelles) rapportées à la distance parcourue en fonction d'un temps adimensionné. La variable de temps adimensionné représente le rapport entre une caractéristique temporelle de la loi (généralement la durée du mouvement) et la période naturelle du mode propre considéré. Ce graphe est une extension d'une représentation utilisée dans [7] destinée à retranscrire les vibrations résiduelles.

\subsection{Graphe de vibration de la loi de référence à accélération limitée}

La loi à accélération limitée, décrite à la figure 3, est utilisée classiquement par toutes les commandes numériques. De par son caractère générique et sa capacité à définir le profil de commande en temps minimum (compte tenu des limitations de vitesse et d'accélération), la loi en bang-bang d'accélération nous servira de 


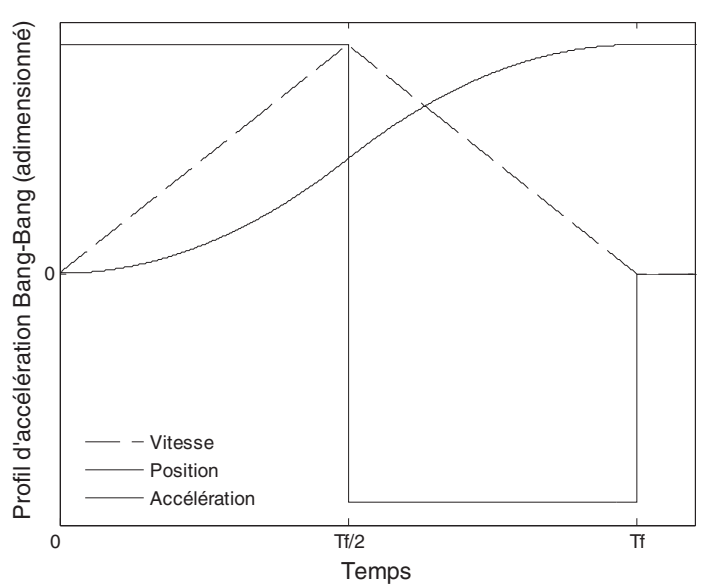

Fig. 3. Loi de mouvement classique à accélération limitée (bang-bang d'accélération).

référence absolue afin de quantifier l'apport des différentes lois étudiées. Le graphe de vibration sera établi pour chaque loi et comparé à celui de la loi à accélération limitée. Le terme d'amortissement associé aux vibrations mécaniques des machines de production est généralement faible (de l'ordre de 5 à $10 \%$ ) et sera négligé dans la suite. On notera que toutes les lois étudiées peuvent être scindées afin d'inclure des phases à accélération nulle (lorsque la vitesse maximale est atteinte). Mais dans l'optique de conserver un outil de comparaison simple pour toutes les lois de mouvement, ces phases ne seront pas prises en compte dans le graphe de vibration. Ainsi, tous les profils seront définis pour atteindre une position finale avec comme seule contrainte la limite d'accélération.

Le profil d'accélération de référence correspondant à la loi à accélération limitée (avec $A_{\mathrm{m}}$ la valeur de l'accélération maximale) s'écrit :

$$
\begin{array}{ll}
0 \leqslant t \leqslant T_{\mathrm{f}} / 2 & \ddot{x}_{\text {ref }}=A_{\mathrm{m}} \\
T_{\mathrm{f}} / 2 \leqslant t \leqslant T_{\mathrm{f}} & \ddot{x}_{\text {ref }}=-A_{\mathrm{m}} \\
T_{\mathrm{f}} \leqslant t & \ddot{x}_{\text {ref }}=0
\end{array}
$$

Tout échelon imposé à un système du second ordre non amorti crée un dépassement d'amplitude égale à $100 \%$ de l'échelon considéré. Lors du mouvement sur le profil en bang-bang d'accélération, le système est soumis à deux échelons (deux commutations), le premier d'amplitude $A_{\mathrm{m}}$ et le second d'amplitude $2 A_{\mathrm{m}}$. Par conséquent, l'amplitude de l'erreur vibratoire maximale (en position) lors du mouvement sera bornée par :

$$
\max _{t<T_{\mathrm{f}}}(\varepsilon(t))=\frac{3 A_{\mathrm{m}}}{\omega_{n}^{2}}
$$

et le calcul de l'erreur vibratoire résiduelle correspondante (cf. Éq. (5)) conduit à l'expression :

$$
\max _{t \geqslant T_{\mathrm{f}}}(\varepsilon(t))=\frac{4 A_{\mathrm{m}}}{\omega_{n}^{2}}\left(\sin \left(T_{\mathrm{f}} \omega_{n}\right)\right)^{2}
$$

En notant que la distance parcourue est donnée par $x_{\text {ref }}=x_{\text {ref }}\left(T_{\mathrm{f}}\right)=A_{\mathrm{m}} T_{\mathrm{a}}^{2}$ avec $T_{\mathrm{f}}=2 T_{\mathrm{a}}$, il est possible de

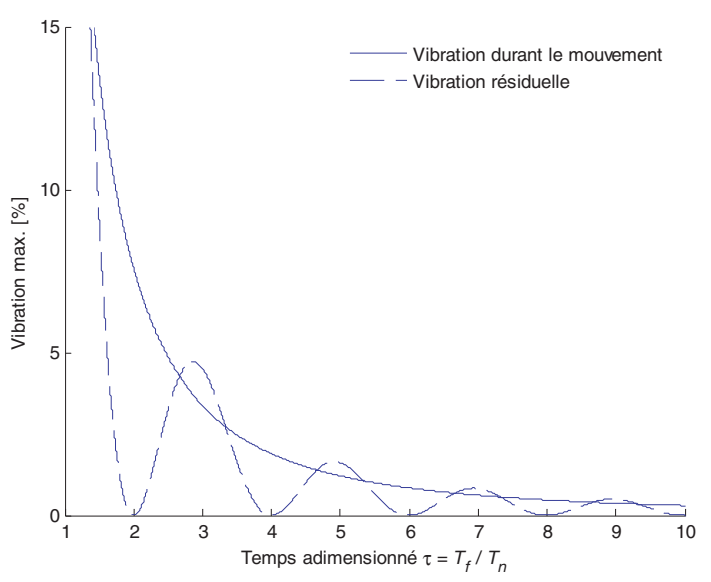

Fig. 4. Graphe de vibration pour la loi à accélération limitée.

reformuler l'amplitude des vibrations sous la forme adimensionnée :

$$
\frac{\max _{t<T_{\mathrm{f}}}(\varepsilon(t))}{x_{\mathrm{ref}}}=\frac{3}{(\pi \cdot \tau)^{2}} ; \quad \frac{\max _{t \geqslant T_{\mathrm{f}}}(\varepsilon(t))}{x_{\mathrm{ref}}}=\left(\operatorname{sinc}\left(\frac{\pi \cdot \tau}{2}\right)\right)^{2}
$$

avec $\tau=T_{\mathrm{f}} \omega_{\mathrm{n}} / 2 \pi$ le temps adimensionné et en adoptant la notation $\operatorname{sinc}(x)=\sin (x) / x$.

Le graphe de vibration de la loi à accélération limitée est représenté à la figure 4. Les spécificités de cette courbe, notamment la présence de zones de vibration résiduelle nulle seront discutées dans la suite. On notera que les vibrations lors du mouvement ne peuvent se développer que pour des durées de mouvement supérieures ou égales à 2 fois la période propre du phénomène vibratoire; en deçà il s'agira d'une déformation élastique de la structure qui n'est ici pas prise en compte.

\section{Les lois de mouvement douces}

\subsection{Les lois harmoniques}

Le déplacement d'un axe d'un point à un autre implique pour une loi de mouvement, l'existence d'une phase d'accélération et d'une phase de décélération. Ce basculement entre des phases où l'accélération sera positive puis négative, laisse tout naturellement penser à la forme périodique des fonctions trigonométriques. A priori, la fonction sinus vérifie les contraintes d'une loi de mouvement. La loi de mouvement en accélération sera ainsi définie par le paramètre d'accélération maximale $A_{\mathrm{m}}$ et la durée du mouvement $T_{\mathrm{f}}$ :

$$
\ddot{x}_{\text {ref }}(t)=A_{\mathrm{m}} \sin \left(2 \pi \frac{t}{T_{\mathrm{f}}}\right) \quad\left(0 \leqslant t \leqslant T_{\mathrm{f}}\right)
$$

On peut imaginer de nombreuses lois de mouvement à base de fonctions trigonométriques; par exemple, la loi en sinus carré d'accélération, disponible dans certaines commandes numériques, dont la pente aux points extrêmes est nulle. On notera que l'utilisation de ce type de loi 


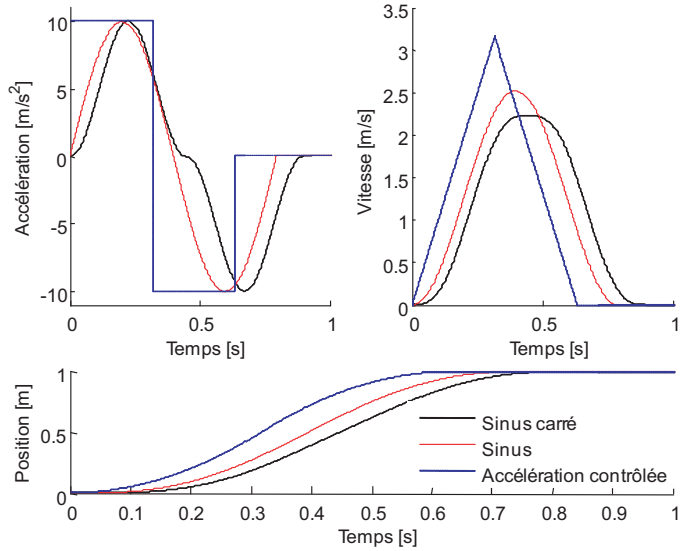

Fig. 5. Loi de mouvement en sinus et sinus carré d'accélération.

rend implicite la discrétisation du profilé; chaque phase du mouvement est définie soit par un sinus carré, soit par une accélération nulle (vitesse constante). Le profil d'accélération pour la loi en sinus carré s'écrit :

$$
\begin{aligned}
& \ddot{x}_{\text {ref }}(t)=A_{\mathrm{m}} \sin ^{2}\left(2 \pi \frac{t}{T_{\mathrm{f}}}\right) \quad\left(0 \leqslant t \leqslant \frac{T_{\mathrm{f}}}{2}\right) \\
& \ddot{x}_{\text {ref }}(t)=-A_{\mathrm{m}} \sin ^{2}\left(2 \pi \frac{t}{T_{\mathrm{f}}}\right) \quad\left(\frac{T_{\mathrm{f}}}{2} \leqslant t \leqslant T_{\mathrm{f}}\right)
\end{aligned}
$$

La figure 5 illustre les lois de mouvement en sinus et sinus carré comparées à la loi à accélération limitée. La figure 6 représente le graphe de vibration associé aux lois trigonométriques. La réduction du spectre d'excitation lors du mouvement par l'utilisation d'une loi de type harmonique est nettement visible. Quant à la réduction des vibrations résiduelles, elle n'est réellement significative que pour des mouvements d'une durée supérieure à 3 ou 4 fois la période propre du mode prépondérant.

\subsection{Les lois polynomiales}

Afin de privilégier la « douceur » d'un mouvement, il semble cohérent de vouloir minimiser les amplitudes des dérivées de la position tout au long du mouvement. Soit $x(t)$ une loi de mouvement pour laquelle nous voulons minimiser la dérivée $n^{\text {ième }}$. Le problème d'optimisation sans contrainte ainsi défini peut se formuler sous la forme quadratique classique : déterminer $x(t)$ minimisant la fonctionnelle $J$ donnée par

$$
J=\int_{0}^{T_{\mathrm{f}}}\left(\frac{\mathrm{d}^{n} x(t)}{\mathrm{d} t^{n}}\right)^{2} \mathrm{~d} t
$$

La solution de ce problème est un polynôme d'ordre $2 n-1$, dont les coefficients seront fonction des conditions aux points extrêmes [8]. Afin de comparer l'influence de la dérivée minimisée, nous allons étudier deux lois de mouvement : la première minimisant le jerk et la seconde minimisant la dérivée du jerk (le snap). Ainsi, la loi minimisant

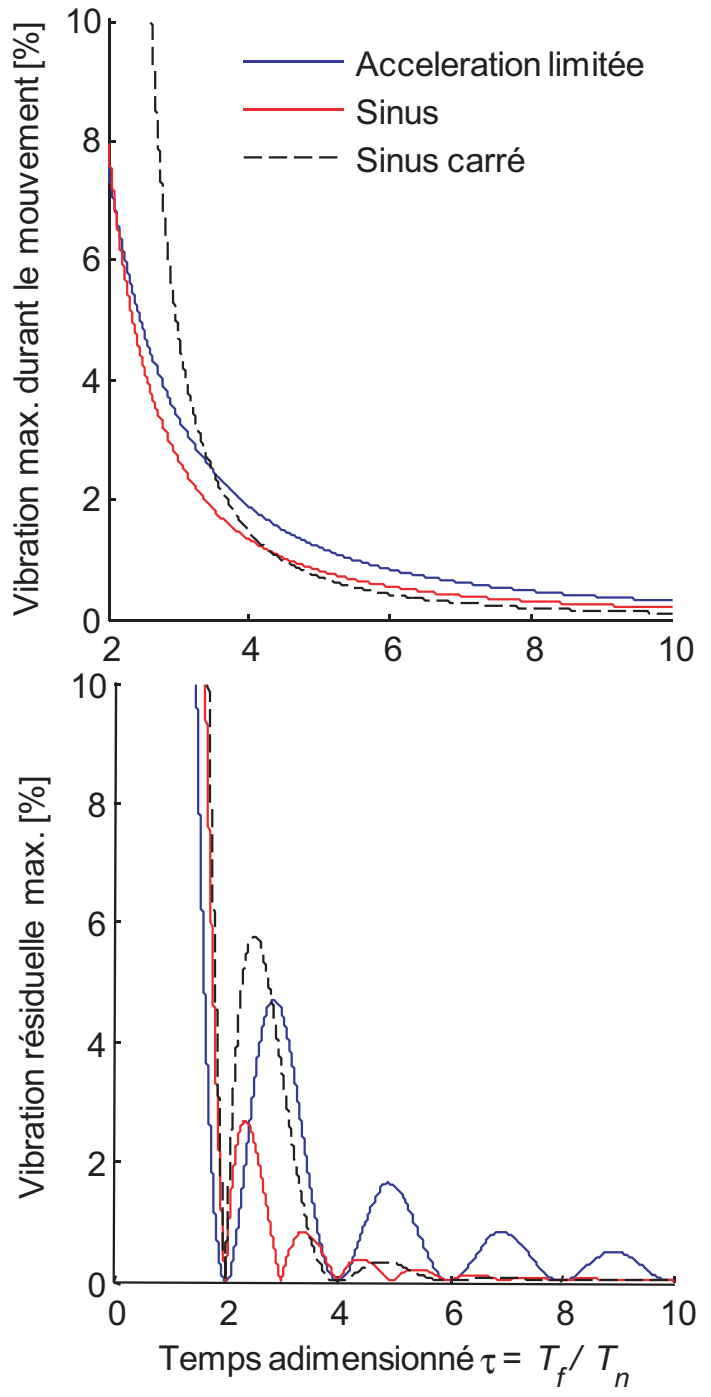

Fig. 6. Graphes de vibration des lois en sinus et sinus carré d'accélération.

le jerk (i.e. pour $n=3$ ), est définie par un polynôme de degré 5 :

$$
\left[x_{\mathrm{ref}}(t)\right]_{\min J}=\frac{30 t^{3} x_{\mathrm{ref}}}{T_{\mathrm{f}}^{5}}\left(\frac{t^{2}}{5}-\frac{T_{\mathrm{f}} t}{2}+\frac{T_{\mathrm{f}}^{2}}{3}\right)
$$

et pour le polynôme minimisant le snap, on obtient :

$$
\left[x_{\mathrm{ref}}(t)\right]_{\min S}=\frac{t^{4} x_{\mathrm{ref}}}{T_{\mathrm{f}}^{7}}\left(-20 t^{3}+70 t^{2} T_{\mathrm{f}}-84 t T_{\mathrm{f}}^{2}+35 T_{\mathrm{f}}^{3}\right)
$$

La figure 7 présente de façon générique la forme de ces lois de mouvement et la figure 8 représente le graphe de vibration associé. L'utilisation des polynômes apporte une nette réduction du niveau vibratoire pour des déplacements d'une durée supérieure à deux fois la période propre du mode dominant. On vérifie que plus le polynôme est d'un degré élevé, donc plus la loi de mouvement est d'une classe de continuité élevée, et moins les vibrations résiduelles sont importantes. La réduction des 

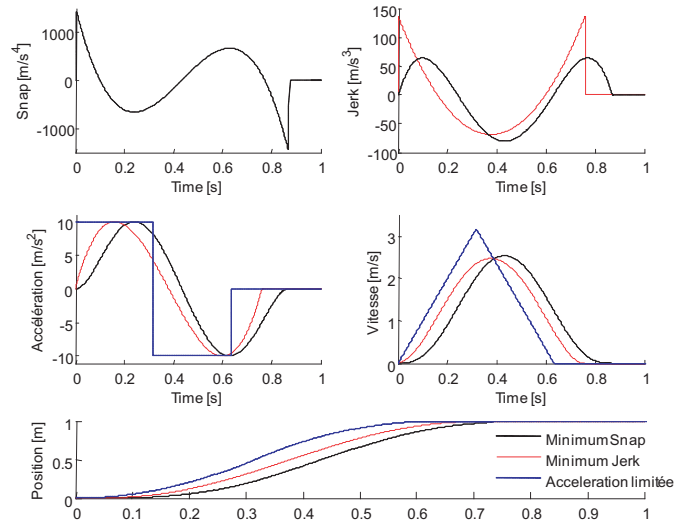

Fig. 7. Loi de mouvement à accélération, jerk et snap minimum.

vibrations lors du mouvement est quant à elle comparable pour les lois à jerk et snap minimum. Il est intéressant de noter que la loi à jerk minimum est utilisée depuis longtemps en robotique pour sa similitude avec les mouvements des articulations humaines [9].

\section{Lois de mouvement basées sur des commutations d'échelons : bang-bang}

\subsection{Définition}

Les lois de mouvement à commutations bang-bang utilisées dans les directeurs de commandes numériques sont des lois de commande en temps optimale imposant une contrainte sur une ou plusieurs dérivées du mouvement. L'optimalité de ces lois suppose un comportement rigide du système, qui est alors assimilable à un double intégrateur. Le problème de commande optimale correspondant peut s'énoncer comme suit :

Soit le système décrit par les équations d'état

$$
\dot{x}_{1}=x_{2}, \dot{x}_{2}=u, \dot{u}=u_{1}, \cdots, \dot{u}_{n-1}=u_{n}
$$

Déterminer l'entrée de commande $u(t)$ qui minimise la fonctionnelle

$$
J=\int_{0}^{T_{\mathrm{f}}} \mathrm{d} t=T_{\mathrm{f}}
$$

avec une contrainte sur la dérivée $n^{\text {ième }}$ de la commande

$$
\left|u_{n}\right| \leqslant\left(u_{n}\right)_{\max }
$$

et les conditions aux limites

$$
\begin{aligned}
x_{1}(0) & =0, x_{1}\left(T_{\mathrm{f}}\right)=x_{\mathrm{ref}}, x_{2}(0)=0, \\
x_{2}\left(T_{\mathrm{f}}\right) & =0, u(0)=0, u\left(T_{\mathrm{f}}\right)=0
\end{aligned}
$$

D'après le principe du maximum de Pontriaguine, la solution optimale vérifie [4] :

(1) la commande optimale est de type bang-bang. $u_{n}$ commute entre les valeurs 0 et $\pm\left(u_{n}\right)_{\max },(2)$ il y a

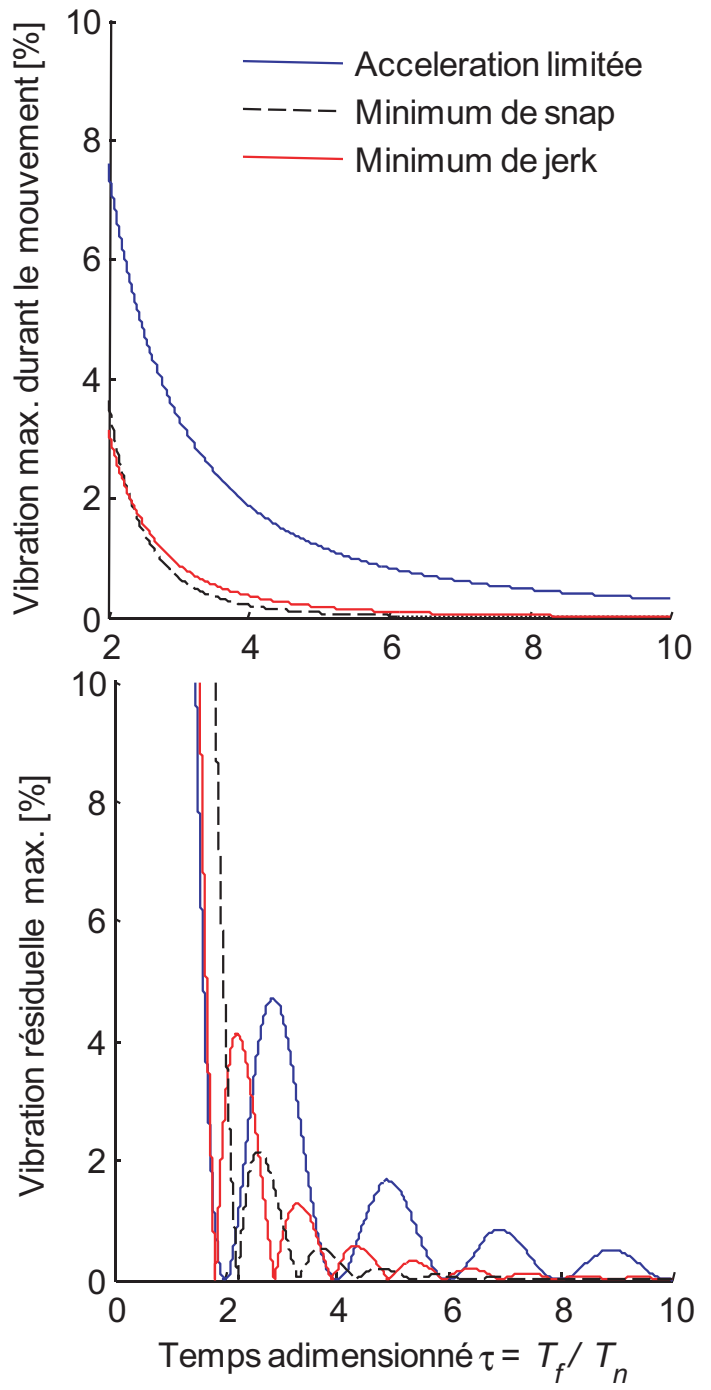

Fig. 8. Graphes de vibration des lois de mouvement à accélération, jerk et snap minimum.

$n+1$ commutations et (3) les instants de commutations sont fonction des conditions aux limites.

L'application de ce résultat pour une limitation d'accélération permet de retrouver la loi en bang-bang d'accélération utilisée comme référence (3 commutations). On note que si l'on ajoute des contraintes sur les autres $n-1$ dérivées de la position, il est alors nécessaire de gérer des paliers de saturation des dérivées concernées. Par exemple, si l'on impose une limitation de vitesse à la loi en bang-bang d'accélération, on obtient un profil en trapèze de vitesse. La figure 9 représente les profils des lois de mouvement en bang-bang de jerk (4 commutations) et en bang-bang de snap ( 5 commutations). On rappelle que pour des raisons de cohérence toutes les lois sont adaptées pour atteindre la même limite d'accélération et que la limitation de vitesse est supposée non atteinte.

D'après les graphes de vibrations de la figure 10, les lois en jerk et snap limités permettent naturellement de réduire les vibrations durant le mouvement. Cette réduction est toutefois moins importante que celle 

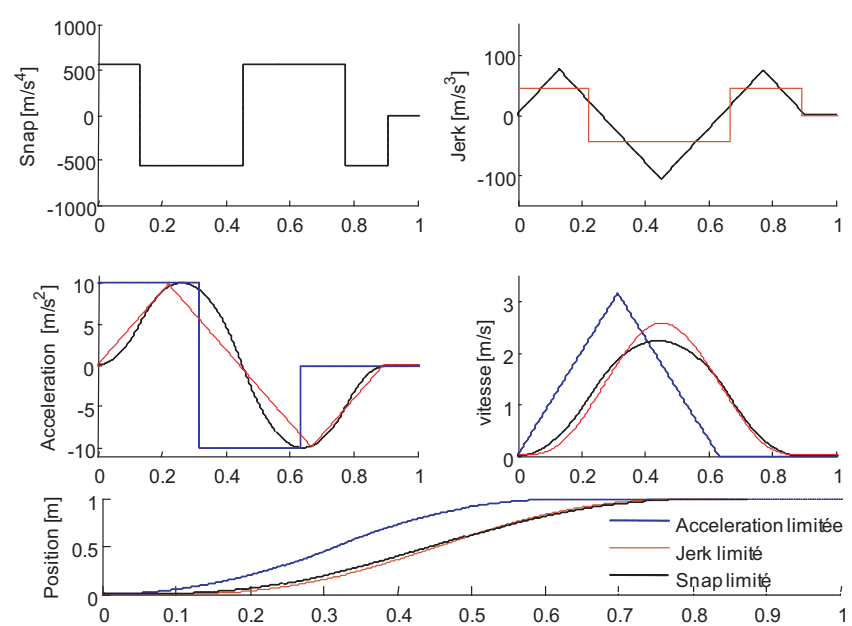

Fig. 9. Profil des lois de mouvement à accélération, jerk et snap limités.

obtenue pour les lois douces polynomiales. La réduction des vibrations résiduelles pour la loi en jerk limité, disponible dans la plupart des commandes du marché, est quant à elle comparable à la celle de la loi à jerk limité. Un aspect notable des lois à commutation bang-bang est l'annulation des vibrations résiduelles pour une durée du mouvement égale à un multiple de deux fois la période du mode propre (à partir de $\tau=4$ pour la loi à jerk limité et $\tau=6$ pour la loi à snap limité). Cette propriété appliquée à la loi à jerk limité peut être utilisée pour définir une procédure de réglage du jerk maximal conduisant à annuler la vibration en fin de mouvement.

\section{2 À propos de l'annulation des vibrations résiduelles}

Pour tous les graphes de vibration présentés, on note l'existence de zones de vibration résiduelle nulle. Ces zones ne sont pas exploitables dans le cas des lois douces. En effet, la durée du mouvement, et donc la variable adimensionnée $\tau=T_{\mathrm{f}} / T_{\mathrm{n}}$, est fixée par la distance à parcourir et par la limitation de l'accélération. Les zones exemptes de vibration ne sont atteintes que pour certaines longueurs de déplacement. Seule la discrétisation du mouvement des lois continues par partie offre le paramétrage nécessaire à l'utilisation de ces zones. Il est possible d'adapter cette propriété aux lois bang-bang vues précédemment.

La capacité des lois à commutation bang-bang d'annuler les vibrations résiduelles par un choix judicieux des durées séparant les instants de commutations est basée sur le même principe que la technique baptisée « Input Shaping Technique ». Cette technique repose sur le fait qu'une vibration engendrée par une impulsion peut être annulée par une vibration causée par une impulsion suivante. L'IST a été étudiée et appliquée par de nombreux chercheurs $[5,10]$. Les amplitudes $A_{i}$ des impulsions et les valeurs $T_{i}$ des instants de commutation sont obtenues à partir de la pulsation naturelle et de l'amortissement du mode propre considéré. Une explication succincte de
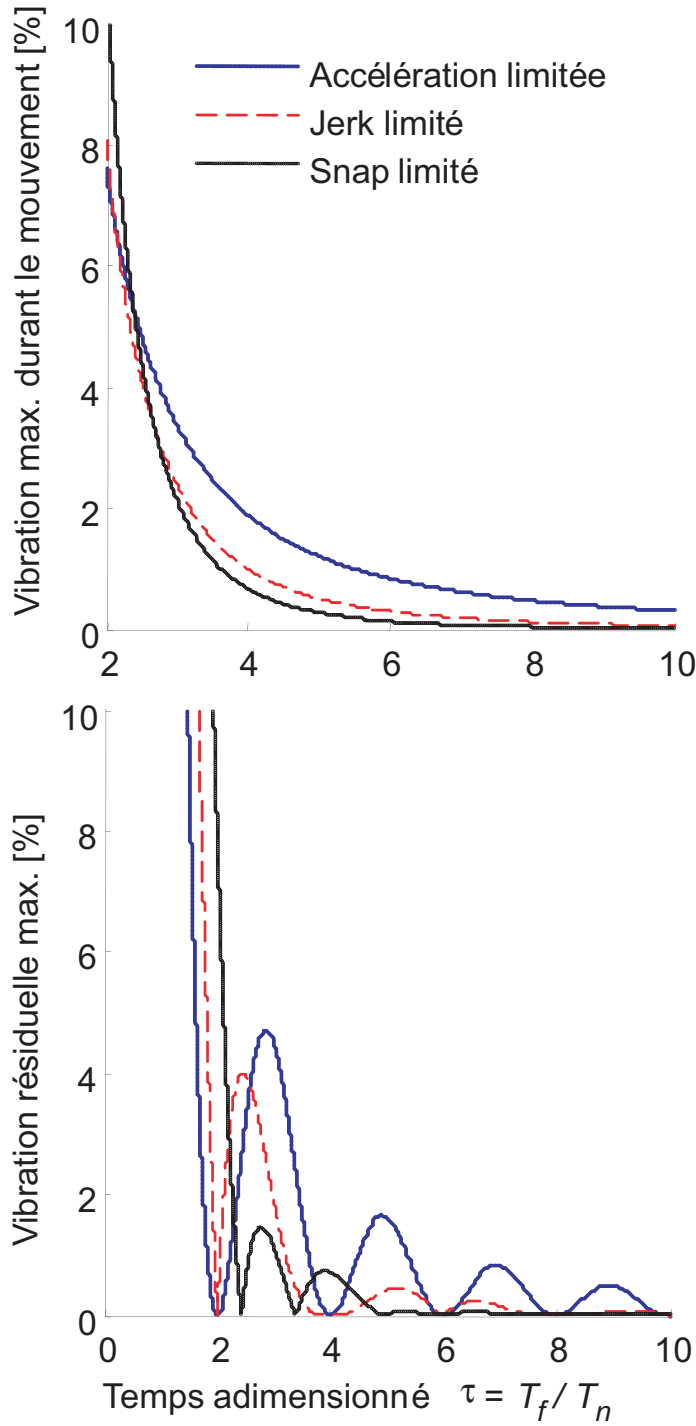

Fig. 10. Graphes de vibration des lois de mouvement à accélération, jerk et snap limités.

ce principe repose sur l'annulation de pôles complexes conjugués réalisée par la loi bang-bang qui est, dans le domaine continu, équivalente à une somme d'échelons retardés dans le temps. Pour exemple, les lois à commutation bang-bang vues précédemment peuvent s'écrire :

$$
\begin{aligned}
& x_{\mathrm{ref}}(s)=\frac{\left(u_{n}\right)_{\max }}{s} \cdot F(s) \cdot \frac{1}{s^{n}} \\
& F(s)=\sum_{i=1}^{n} A_{i} \cdot e^{-s T_{i}}
\end{aligned}
$$

avec $F(\mathrm{~s})$ un filtre retardé et les $A_{i}$ prennent ici leur valeur dans l'ensemble $\{0,1,-1\}$. L'annulation de pôles complexes conjugués associés au mode vibratoire s'obtient par la présence de zéros complexes conjugués à la même position dans le filtre retardé :

$$
\left.\sum_{i=1}^{n} A_{i} \cdot e^{-s T_{i}}\right|_{s=-\varsigma_{n} \omega_{n} \pm j \omega_{n} \sqrt{1-\varsigma_{n}^{2}}}=0
$$




$$
\begin{aligned}
& I_{\mathrm{vib}}=1-\frac{\text { Aire sous le graphe de vibration }}{\text { Aire sous le graphe de vibration pour la loi de référence }} \\
& I_{\mathrm{vibres}}=1-\frac{\text { Aire sous le graphe de vibration résiduelle }}{\text { Aire sous le graphe de vibration résiduelle pour la loi de référence }}
\end{aligned}
$$

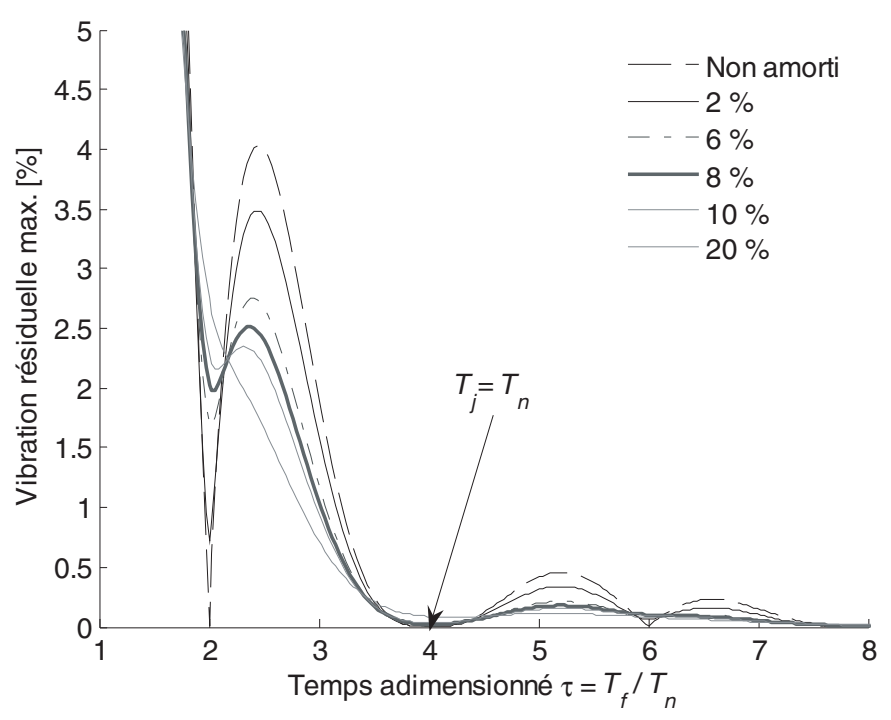

Fig. 11. Graphes de vibration résiduelle de la loi à jerk limité pour différentes valeurs d'amortissement du mode vibratoire.

Cette équation implique que les deux conditions suivantes soient indépendamment vérifiées :

$$
\left\{\begin{array}{l}
\sum_{i=1}^{n} A_{i} \cdot e^{\varsigma_{n} \omega_{n} T_{i}} \cos \left(\omega_{n} \sqrt{1-\varsigma_{n}^{2}} T_{i}\right)=0 \\
\sum_{i=1}^{n} A_{i} \cdot e^{\varsigma_{n} \omega_{n} T_{i}} \sin \left(\omega_{n} \sqrt{1-\varsigma_{n}^{2}} T_{i}\right)=0
\end{array}\right.
$$

L'IST utilise le nombre de commutation $n$, l'amplitude des impulsions $A_{i}$ et les instants de commutations $T_{i}$ comme variables de résolutions pour l'équation (21). Dans notre cas, les amplitudes et le nombre de commutation sont imposés par le mouvement à réaliser et par la forme de loi considérée. Les seuls paramètres libres sont les instants de commutation (la durée du mouvement). On notera que c'est la raison pour laquelle les lois bang-bang précédentes ne permettent d'annuler totalement les vibrations résiduelles que dans le cas d'un mode non amorti. Si l'amortissement n'est pas négligeable, l'amplitude des impulsions doit alors être adaptée. Considérons la loi à jerk limité (cf. Fig. 9) appliquée à un mode non amorti. En notant, $J_{\mathrm{m}}$ la valeur maximale du jerk et $T_{\mathrm{j}}$ la durée des échelons de jerk, le système d'équations (21) se réécrit :

$$
\left\{\begin{array}{l}
J_{m}\left(1-2 \cos \left(\omega_{n} T_{\mathrm{j}}\right)+2 \cos \left(3 \omega_{n} T_{\mathrm{j}}\right)-\cos \left(4 \omega_{n} T_{\mathrm{j}}\right)\right)=0 \\
J_{m}\left(-2 \sin \left(\omega_{n} T_{\mathrm{j}}\right)+2 \sin \left(3 \omega_{n} T_{\mathrm{j}}\right)-\sin \left(4 \omega_{n} T_{\mathrm{j}}\right)\right)=0
\end{array}\right.
$$

Une solution triviale consiste à choisir la durée des échelons de jerk égale à la période propre $T_{\mathrm{n}}\left(=2 \pi / \omega_{n}\right)$ du mode vibratoire. Dans ce cas, la valeur maximale du jerk sera fixée par la limite d'accélération :

$$
J_{m}=A_{\mathrm{m}} / T_{\mathrm{n}}
$$

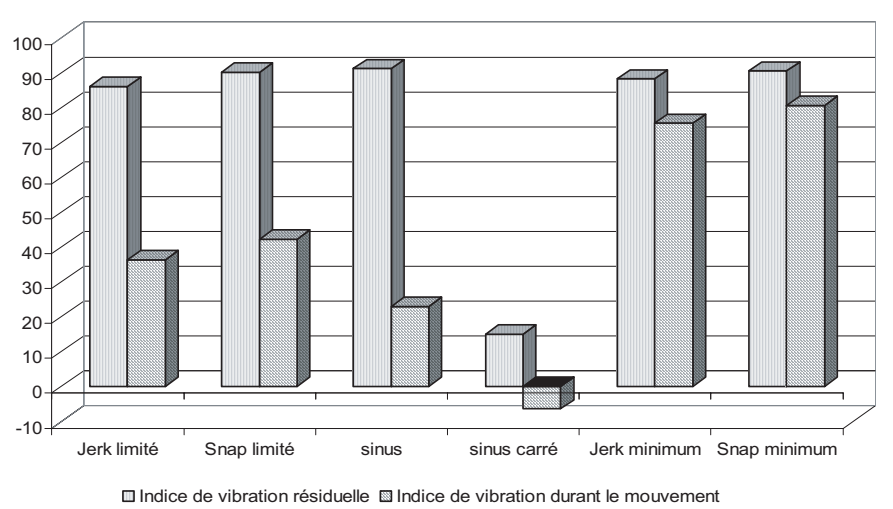

Fig. 12. Indices de performance des lois de mouvement.

et la durée du mouvement sera au minimum de $4 T_{\mathrm{n}}$.

On notera que la loi à jerk limité étant symétrique par rapport à la demi-durée de mouvement, le résultat précédent reste vrai même dans le cas d'un profil incluant la limitation de vitesse. On notera également que l'amortissement des systèmes mécaniques étant très faible, la réduction des vibrations reste largement notable dans le cas d'un mode amorti [11]. La figure 11 illustre cet effet en présentant le graphe de vibration résiduelle de la loi à jerk limité pour différentes valeurs d'amortissement.

\section{Indices de performances des lois de mouvement}

Afin de quantifier les performances des différentes lois de mouvement étudiées, deux indices de performance sont déduits du graphe de vibration : un indice de vibration lors du mouvement $I_{\mathrm{vib}}$ et un indice de vibration résiduelle $I_{\text {vibres }}$ définis comme suit :

$$
\text { voir équation (24) ci-dessus }
$$

La figure 12 présente l'évolution de ces critères en fonction du type de loi considéré. Le tableau 1 donne l'allongement de la durée de mouvement théorique pour ces différentes lois. On remarque en comparant les différents indices de performance que les lois en bang-bang de jerk ou de snap peuvent permettre de réaliser un meilleur compromis vis-à-vis de la réduction des oscillations résiduelles et de l'inévitable allongement de la durée de mouvement théorique (par rapport au bang-bang d'accélération). Il s'agit de plus d'indice de performance réalisant une moyenne. Or nous venons de vérifier que les lois en bangbang peuvent annuler les vibrations résiduelles pour un réglage judicieux de la durée séparant les échelons. Les lois polynomiales à jerk ou à snap minimum réduisent de façon plus significative les vibrations lors du mouvement, mais ceci au détriment du temps de cycle. Quant 
Tableau 1. Augmentation de la durée du mouvement pour les lois étudiées.

\begin{tabular}{l|l}
\hline \hline $\begin{array}{l}\text { Type de loi } \\
\text { de mouvement }\end{array}$ & $\begin{array}{l}\text { Augmentation de la durée } \\
\text { du mouvement théorique par rapport } \\
\text { à la loi de référence }\end{array}$ \\
\hline Jerk limité & $\begin{array}{l}\text { Selon la valeur du jerk maximum } \\
\text { entre 0 et }+41 \%\end{array}$ \\
\hline Snap limité & $\begin{array}{l}\text { Selon la valeur du jerk et snap maximum } \\
\text { entre } 0 \text { et }+44 \%\end{array}$ \\
\hline Sinus & $+25 \%$ \\
\hline Sinus carré & $+41 \%$ \\
\hline Jerk minimum & $+20 \%$ \\
\hline Snap minimum & $+37 \%$ \\
\hline
\end{tabular}

aux lois harmoniques étudiées, elles n'améliorent pas un critère spécifique et ne permettent pas plus de réaliser un compromis satisfaisant entre les trois critères.

\section{Validations expérimentales}

Dans cette partie, nous nous proposons d'illustrer l'influence des lois de mouvement étudiées par quelques validations expérimentales. Pour ce faire nous employons un banc mono-axe équipé d'un moteur linéaire synchrone Indramat LSP120C. Ce type de machine est utilisé pour déplacer rapidement un système d'usinage (tête de perçage). Afin de reproduire le comportement dynamique du système embarqué utilisé en production, deux ensembles masses/ressorts peuvent être ajoutés en série sur la table en translation (cf. Fig. 13). Un des ensembles masse-ressort est ici bloqué mécaniquement afin de ne tenir compte que d'un mode de déformation unique dominant la réponse vibratoire du système. La fréquence propre associée à ce mode est de $12,1 \mathrm{~Hz}$ $\left(76 \mathrm{rad} . \mathrm{s}^{-1}\right)$. Le système correspond ainsi exactement aux hypothèses de travail considérées dans cet article. Les relevés expérimentaux présentés sont par conséquent une illustration directe de l'influence des lois de mouvement.

Les différentes lois étudiées sont calculées pour respecter l'accélération et la vitesse maximale sur l'axe concerné. Ces données associées à la distance à parcourir suffisent pour élaborer la loi de référence à accélération limitée, ainsi que les lois polynomiales et trigonométriques. En ce qui concerne les lois à jerk ou snap limité, nous utilisons la méthode de réglage décrite dans la section 4.2 visant à supprimer les vibrations résiduelles. Ainsi, pour la loi à jerk limité, la valeur maximal de jerk est fixée par l'accélération maximale et par la période propre du système. Pour la loi à snap limité, la valeur de snap maximal est fixée par le jerk maximal et par la période propre du système. Par contre, il n'existe pas de limite théorique à la valeur du jerk dans ce cas de figure. Nous avons par conséquent décliné deux cas de réglage : un premier cas où nous utilisons la même valeur de jerk maximal que

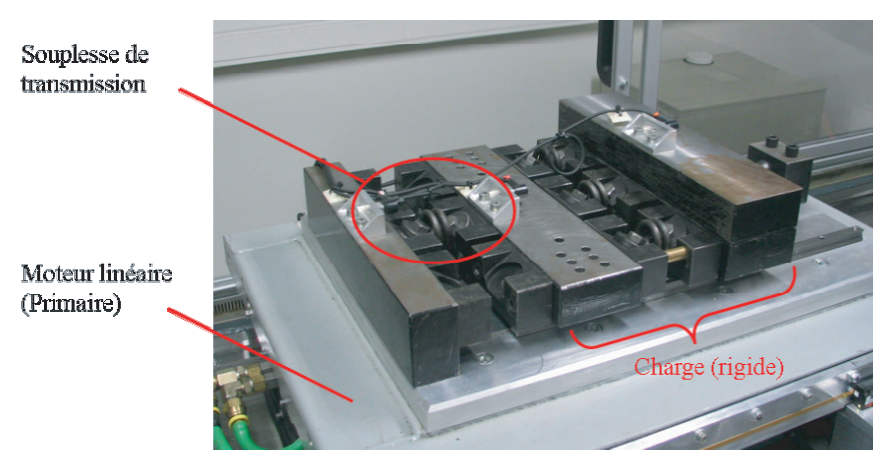

Fig. 13. Banc d'essais mono-axe. (Caractéristiques : masses embarquées $260+(20,6+16,5) \mathrm{kg}$; accélération $=20 \mathrm{~m} \cdot \mathrm{s}^{-2}$; vitesse $=100 \mathrm{~m} \cdot \mathrm{min}^{-1}$.)

pour la loi à jerk limité, un deuxième où la valeur de jerk est le double de la précédente.

La figure 14 présente les résultats obtenus. Les essais ont été réalisés pour plusieurs longueurs de déplacement. Par souci de clarté, les courbes présentées correspondent à un déplacement de faible amplitude $(200 \mathrm{~mm})$ permettant une mise en évidence plus nette des performances en termes de réduction de vibration (l'amortissement du mode n'a que peu d'influence dans ce cas puisque les vibrations induites par les différentes phases du mouvement n'ont pas le temps de s'atténuer). La figure 14 illustre le fait que la procédure de réglage utilisée pour les lois à jerk et snap limités permet effectivement de réduire considérablement les vibrations résiduelles. On notera que dans le cas de la loi à snap limité, la valeur du jerk maximal influe sur l'erreur résiduelle. Pour un jerk double de celui réglé pour la loi à jerk limité, les vibrations résiduelles augmentent légèrement et la durée de mouvement reste évidemment toujours supérieure à celle obtenue pour la loi à jerk limité. Pour un jerk maximum équivalent sur les deux lois, la loi à snap limité ne provoque pas d'oscillations résiduelles perceptibles. Concernant les lois polynomiales, l'augmentation du degré du polynôme accroît naturellement sa « douceur » et l'on vérifie sur les courbes expérimentales que les vibrations diminuent significativement avec cette augmentation. Les résultats obtenus avec les lois en sinus et sinus carré d'accélération montrent également une nette réduction des vibrations durant le mouvement comparativement à la loi de référence.

\section{Conclusions}

Dans cet article nous avons présenté une analyse comparative de différentes lois de mouvement (lois bangbang, lois harmoniques et lois polynomiales) sur les vibrations et sur l'allongement de la durée de mouvement. La méthode d'obtention d'un majorant de l'erreur vibratoire a été présentée. Les résultats de cette formalisation, traduits sous la forme de graphes de vibration, sont détaillés pour chacune des lois. Des indices de performance établis 

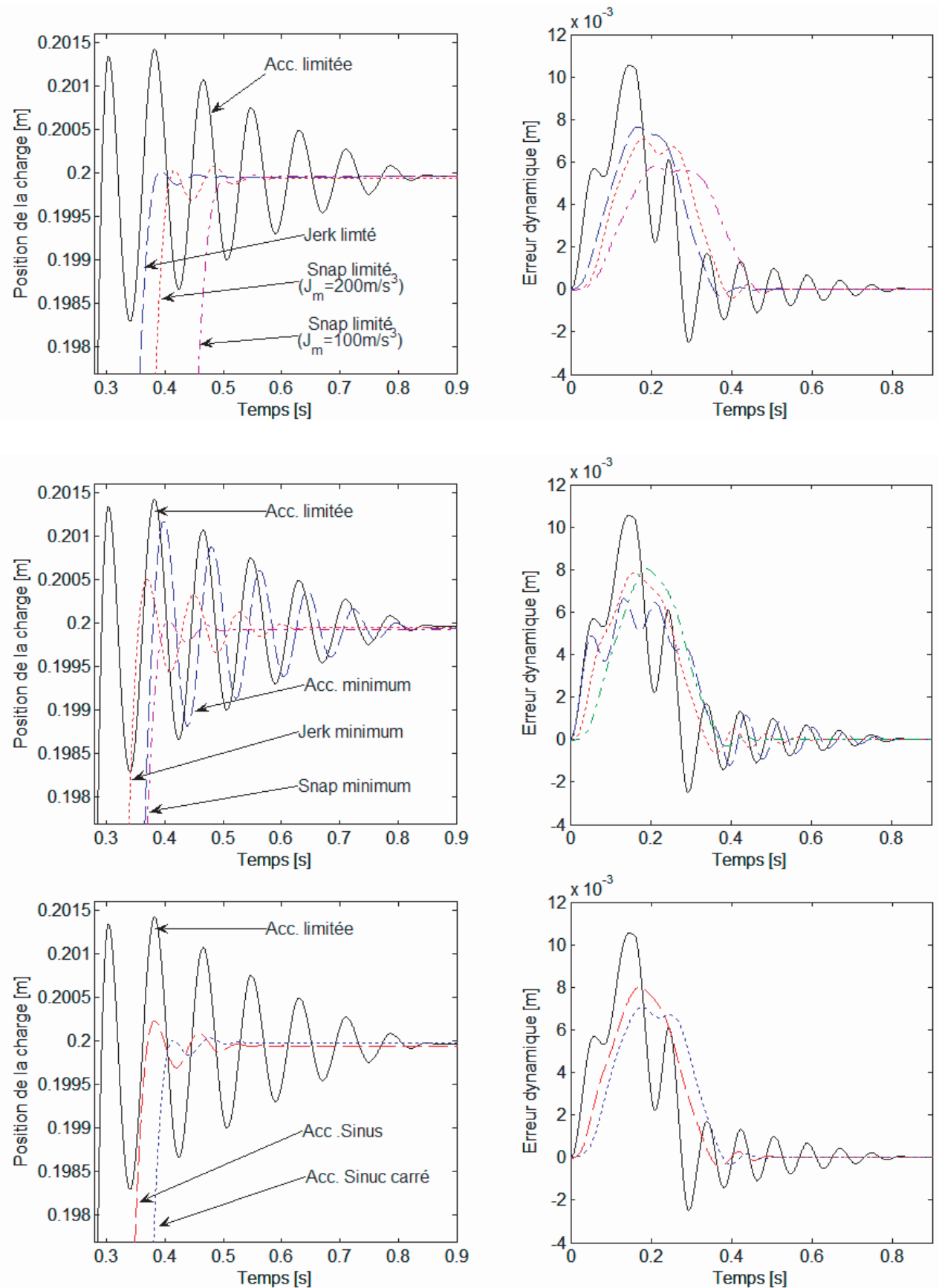

Fig. 14. Vibrations résiduelles et erreur de poursuite des lois de mouvement étudiées (mouvement de $200 \mathrm{~mm}$ ).

à partir de ces mêmes graphes permettent d'analyser a priori la propension d'une loi de mouvement à améliorer les performances du positionnement. De cette analyse peut découler le choix d'un certain type de lois en fonction du contexte d'utilisation du système. Pour les machines réalisant des opérations du type pick-and-place ou pour les usinages où le temps est le critère privilégié (automobile/aéronautique), la famille des lois bang-bang offre la meilleure solution. Pour des opérations où la précision du suivi de trajectoire est seule privilégiée, il semble préférable de recourir à la douceur « polynomiale ».
Il est intéressant de noter que deux lois de mouvement se dégagent principalement. Il s'agit des lois en jerk (jerk limité et jerk minimum) qui apparaissent comme les mieux adaptées au cas d'un mode vibratoire dominant. La première permet d'annuler facilement les vibrations résiduelles mais nécessite une certaine connaissance du phénomène vibratoire, la seconde réduit notablement les vibrations pour tout type de déplacement, confirmant ainsi son aptitude à décrire les mouvements humains naturellement doux (les modes vibratoires des articulations humaines sont très bas). 


\section{Références}

[1] P. Van Den Braembussche, P. De Fonseca, H. Van Brussel, P. Sas, Active control of machine tool flexibility, Proceedings of MOVIC'98, ETH Zurich, Suisse, 1998

[2] D. Renton, M.A. Elbestawi, High speed servo control of multi-axis machine tools, Int. J. Machine Tool Manufacture 40 (2000) 539-559

[3] J.B. Dingwell, Ch.D. Mah, F.A. Mussa-Ivaldi, Experimentally Confirmed Mathematical Model for Human Control of a Non-Rigid Object, J. Neurophysiology 91 (2004) 1158-1170

[4] T.D. Tuttle, Creating time-optimal commands for linear systems, Thèse, département d'ingénierie mécanique du M.I.T, 1997

[5] W. Singhose, L. Porter, M. Kenison, E. Kriikku, Effects of hoisting on the input shaping control of gantry cranes, Control Engineering Practice 8 (2000) 1159-1165

[6] R. Béarée, P.-J. Barre, S. Bloch, Influence of highspeed machine tool control parameters on the contouring accuracy - Application to linear and circular interpolation, J. Intelligent Robotic Systems 40 (2004) 321-342

[7] M.W.M.G. Dissanayake, A.N. Poo, Robot trajectory planning for minimising residual vibrations, Proc. IEEE Int. Symp. Intelligent Control, 1988, pp. 471-474

[8] D. Brun-Picard, Influence des lois de mouvement sur les déformations et les vibrations des machines à grande vitesse, Proc. $3^{\text {e }}$ Assises MO\&UGV, 2004, pp. 81-90

[9] C.M. Harris, Exploring smoothness and discontinuities in human motor behaviour with fourier analysis, Mathematical biosciences 188 (2004) 99-116

[10] N.C. Singer, W.P. Seering, Design and Comparison of Command Shaping Methods for Controlling Residual Vibration, Proc. IEEE Int. Conf. Robotics and Automation, Scottsdale, AZ, 1989, pp. 888-893

[11] R. Béarée, P.-J. Barre, P. Borne, E. Dumetz, Influence of a Jerk Controlled Movement Law on the Vibratory Behaviour of High-Dynamics Systems, J. Intelligent Robotic Systems 42 (2005) 275-293 